\title{
Note on the Luminosity Distance
}

L. N. K. DE Silva

Department of Mathematics, University of Colombo, Colombo, Sri Lanka.

(Date of receipt : 23 August 1982)

(Date of acceptance : 09 December 1982)

\begin{abstract}
In the literature we find that the luminosity distance of a source is obtained in the frame of the source and not in the frame of the observer. It is proposed here that the luminosity distance should be obtained in the frame of the observer for it to be physically meaningful. A necessary conclusion that follows is that the estimated absolute luminosities of the quasars are less than the currently accepted values.
\end{abstract}

\section{Introduction}

The absolute luminosities of distant sources like quasars can be inferred if their apparent luminosities and the luminosity distances are known. The apparent luminosities are measured on the earth and the luminosity distances are given in terms of the redshift of the source.

In the literature (for example Mc Vittie ${ }^{1}$ and Weinberg ${ }^{2}$ ) the expression for the luminosity distance is obtained as follows. In the Robertson Walker metric;

$$
d s^{2}=c^{2} d t^{2}-\frac{R^{2}(t)}{\left(1+\frac{k r^{2}}{4}\right)^{2}}\left[d r^{2}+r^{2} d \theta^{2}+r^{2} \sin ^{2} \theta d \phi^{2}\right]
$$

the apparent luminosity of a source $\mathrm{S}$ with absolute luminosity $\mathrm{L}$ is given as,

$$
\frac{\mathrm{L}\left(1+\frac{k r_{i}{ }^{2}}{4}\right)^{2}}{4 \pi R_{o}{ }^{2}(1+z)^{2} r_{i}{ }^{2}}
$$

where $R_{0}$ is the value of scale factor $R(t)$ at $t_{0}$ the time of observation of light emitted by $S, z$ and $r_{i}$ are the redshift and the comoving radial coordinate of $S$.

The factor $(1+z)^{2}$ in the above expression is due to the redshift of the energy and the change in the time intervals of emission and arrival of the photons.

$$
\frac{4 \pi R_{0}^{2} r_{i}^{2}}{\left(1+\frac{k r_{i}^{2}}{4}\right)^{2}} \begin{aligned}
& \text { is the area of the pseudo - sphere } \mathrm{r}_{i}=\text { constant with centre at the } \\
& \text { source at time } \mathrm{t}_{\mathrm{o}} .
\end{aligned}
$$




\section{Discussion}

However the question arises whether we are justified in determining the area of the pseudo-sphere with centre at the source. My contention is that the luminosity distance thus obtained is the distance measured in the frame of the source. The area of the pseudo-sphere should be,

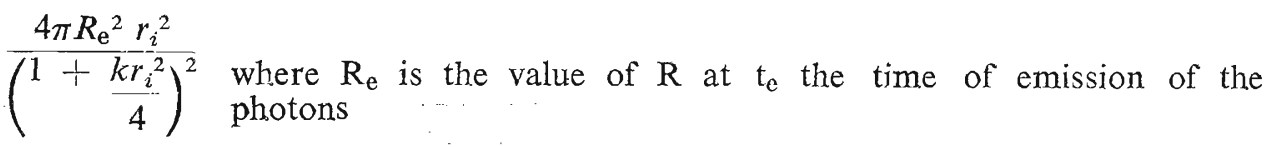

One might say that the universe expands and therefore photons have to travel a greater distance. But then the question may be asked as to in which frame this distance is measured.

This can be made clear by considering the Milne model whose metric can be obtained by a co-ordinate transformation from the metric in special relativity.

Writing $r=2 \tan h \frac{w}{2}, k=-1$ and $R(t)=c t$ in the Robertson-Walker metric we obtain the metric of the Milne model in the form,

$d s^{2}=c^{2} d t^{2}-c^{2} t^{2}\left[d w^{2}+\sin h^{2} w\left(d \theta^{2}+\sin ^{2} \theta d \phi^{2}\right)\right]$

so that $w$ is the comoving radial co-ordinance of an object.

This can be written in the form.

$d s^{2}=c^{2} d \tau^{2}-d \rho^{2}-\rho^{2}\left(d \theta^{2}+\sin ^{2} \theta d \phi^{2}\right)$

by the transformation $\tau=t \cos h w$ and $\rho=c t \sin h w$

A source with the comoving co-ordinate $w$ (constant) in metric (1) at time $t_{\mathrm{e}}$ (say the time of emission of a light ray) is at a distance $\rho_{\mathrm{e}}=c t_{\mathrm{e}} \sin h \mathrm{w}$ and is moving with constant velocity $\tan h w$ with respect to the observer in metric (2). Further the event $\left(w, t_{\mathrm{e}}\right)$ has co-ordinates $\rho_{\mathrm{e}}$ and $\tau_{\mathrm{e}}=t_{\mathrm{e}} \cos h w$ with respect to the metric (2). This is consistent with the interpretation that the source receding away from the observer has moved a distance $c t_{\mathrm{e}} \sin h w$ with velocity $c \tan h w$ in time $t_{\mathrm{e}} \cos h w$ with respect to the observer.

In special relativity the radius of the pseudo-sphere that goes into the calculation of the luminosity distance is $c t_{\mathrm{e}} \sin h w$, in the frame of the observer.'

Now if one uses the expression $\frac{R_{0} r_{i}}{1-\frac{r_{i}^{2}}{4}}$ for the radius as done in the text books and current literature one would arrive at the expression $c t_{0} \sin h w$ where $t_{0}$ is the time of arrival at the observer of a ray of light emitted from the source at time $t_{\mathrm{e}}$ as measured with respect to the metric. (1). This clearly is not the distance measured in the frame of the observer, using the special relativistic metric. 


\section{Conclusion}

In calculating the apparent luminosity, what we are interested is in finding the luminosity of a source in the frame of the observer and not in the frame of the source. In view of the above discussion, it is reasonable to assume that the apparent luminosity of a source with absolute luminosity $\mathrm{L}$ is given by

$$
\frac{L\left(1+\frac{k r_{i}{ }^{2}}{4}\right)^{2}}{4 \pi R_{\mathrm{e}^{2}}{ }^{2}(1+z)^{2} r_{i}{ }^{2}} \text { and not by } \frac{L\left(1+\frac{k r_{i}{ }^{2}}{4}\right)^{2}}{4 \pi R_{0}{ }^{2}(1+z)^{2} r_{i}{ }^{2}}
$$

One important consequence is that a source with a given absolute luminosity at a given red shift will have its apparent luminosity increased by a factor

$$
\left(\frac{R_{0}}{R_{\mathrm{e}}}\right)^{2}=(1+z)^{2}
$$

If the apparent luminosity is given, there is a reduction in the inferred absolute luminosity by a factor $(1+z)^{2}$. For example, the estimated absolute luminosity of a quasar at $z=2.16$ is reduced by a factor of 10 .

\section{Acknowledgement}

I wish to thank Professor V. Joseph for helpful comments and suggestions.

\section{References}

1. MC Virtie, G.C. (1965). General Relativity and Cosmology, second edition. Chapman \& Hall Ltd.

2. Weinberg, S. (1972). Gravitation and Cosmology. John Wiley \& Sons, Inc. 